\section{Integrated intravascular optical coherence tomography ultrasound imaging system}

\author{
Jiechen Yin, ${ }^{a}$ Hao-Chung Yang, ${ }^{b}$ Xiang Li, ${ }^{b}$ Jun Zhang, ${ }^{c}$ \\ Qifa Zhou, ${ }^{b}$ Changhong Hu, ${ }^{b}$ K. Kirk Shung, ${ }^{b}$ and \\ Zhongping Chen ${ }^{\mathrm{a}, \mathrm{c}, *}$ \\ ${ }^{a}$ University of California, Irvine, Department of Biomedical \\ Engineering, Irvine, California 92697 \\ b University of Southern California, NIH Transducer \\ Resource Center and Department of Biomedical Engineering, \\ Los Angeles, California 90089-1111 \\ 'University of California, Irvine, Beckman Laser Institute, \\ 1002 Health Sciences Road East, Irvine, California \\ 92612
}

\begin{abstract}
We report on a dual-modality optical coherence tomography (OCT) ultrasound (US) system for intravascular imaging. To the best of our knowledge, we have developed the first integrated OCT-US probe that combines OCT optical components with an US transducer. The OCT optical components mainly consist of a single-mode fiber, a gradient index lens for light-beam focusing, and a rightangled prism for reflecting light into biological tissue. A 40- $\mathrm{MHz}$ piezoelectric transducer (PZT-5H) side-viewing US transducer was fabricated to obtain the US image. These components were integrated into a single probe, enabling both OCT and US imaging at the same time. In vitro OCT and ultrasound images of a rabbit aorta were obtained using this dual-modality imaging system. This study demonstrates the feasibility of an OCT-US system for intravascular imaging, which is expected to have a prominent impact on early detection and characterization of atherosclerosis. () 2010 Society of Photo-Optical Instrumentation Engineers. [DOI: 10.1117/1.3308642]
\end{abstract}

Keywords: optical coherence tomography; intravascular ultrasound; intravascular imaging; dual-modality imaging system.

Paper 09374LR received Aug. 25, 2009; revised manuscript received Dec. 17, 2009; accepted for publication Dec. 21, 2009; published online Feb. 18, 2010.

\section{Introduction}

Atherosclerosis is one of the major causes of morbidity and mortality in developed countries. It is characterized by thickening of the arterial vessel wall due to atheromatous plaque, which may result in narrowing or even occlusion of arterial lumen. Vulnerable plaques are subject to rupture, causing most cases of myocardial infarction and stroke. ${ }^{1}$ Although the understanding of vulnerable plaques is still at an early stage, previous research has demonstrated that the thin-cap fibroatheroma (TCFA) accounts for $\sim 80 \%$ of sudden cardiac death. ${ }^{2}$ TCFAs have been histologically identified with the following pathological features: thin fibrous cap $(<65 \mu \mathrm{m})$, large lipid

*Address all correspondence to: Zhongping Chen, Ph.D. Tel: 949-824-1247; Fax: 949-824-8413; E-mail: z2chen@uci.edu. pool, and macrophages near or within the fibrous cap. ${ }^{2,3}$ Biomedical imaging techniques, such as angiography, computed tomography, and magnetic resonance imaging, have been used to detect and assess atherosclerotic lesions. ${ }^{4}$ However, all three techniques can only reveal the shape of arterial lumen, which is often deceptive because the lumen area may remain unaffected until the final stages of the disease.

Currently, intravascular ultrasound (IVUS) has been increasingly used in the clinic for the diagnosis of atherosclerosis because it permits direct tomographic visualization of cross-sectional images of the vessel wall. ${ }^{5-7}$ However, the spatial resolution of IVUS is inadequate to distinguish the microstructure of TCFAs, especially an accurate thickness of the thin fibrous cap. On the other hand, optical coherence tomography (OCT) is a newly developed optical-based technology that can provide real-time, high-resolution, threedimensional images with micrometer resolution, which is 1-2 orders better than that of IVUS. ${ }^{8}$ OCT has been performed for intravascular imaging and evaluation of vulnerable plaques. $^{4-9-11}$ The outstanding resolution advantage of OCT over other imaging modalities makes it "the only method demonstrated to be capable of measuring all of the microscopic features associated with TCFAs to date." the major drawback of OCT is its limited penetration depth. It is extremely difficult for OCT to image the whole depth of a large lipid pool within a TCFA that appears as a signal-poor region with an unclear border underlying the thin fibrous cap.

Recently, several research groups have demonstrated that the two imaging modalities provide complementary information for detecting TCFAs. ${ }^{12,13}$ OCT's high resolution enables an accurate thickness measurement of the fibrous cap, and IVUS provides whole penetration depth of the vessel wall. In addition, a minimal amount of flushing agent will be needed for OCT once the area of interest is found by IVUS. Recently, Sawada et al. ${ }^{12}$ studied the feasibility of the combined use of IVUS and OCT data (images acquired separately) for detecting TCFAs within 56 patients. The results clearly showed that neither modality alone was sufficient for detecting TCFAs and that the combined use of OCT and IVUS would significantly increase the sensitivity and specificity for detection of TCFAs.

In this paper, we report on the development of an integrated intravascular OCT-ultrasound (US) (OCT-US) imaging system that combines the high spatial resolution of OCT for accurate plaque characterization and the deep penetration depth of IVUS. In vitro imaging of a normal rabbit aorta was achieved by using this OCT-US system, which demonstrated the integrated system's feasibility for intravascular imaging and potential in diagnosis and characterization of atherosclerotic plaques.

\section{Materials and Methods}

The schematic of the dual-modality probe that combines OCT optical components with an US transducer is shown in Fig. 1. A 0.5-mm-diam gradient index lens (NSG America, Inc., Somerset, NJ) was used to focus light from a single-mode fiber tip, followed by a microprism (Tower Optical Corp., Boynton Beach, FL) reflecting the focused light beam into tissue. These optical components were fixed and sealed in a transparent thin-wall polyertrafluoroethylene tubing (Zeus,

1083-3668/2010/15(1)/010512/3/\$25.00 @ 2010 SPIE 


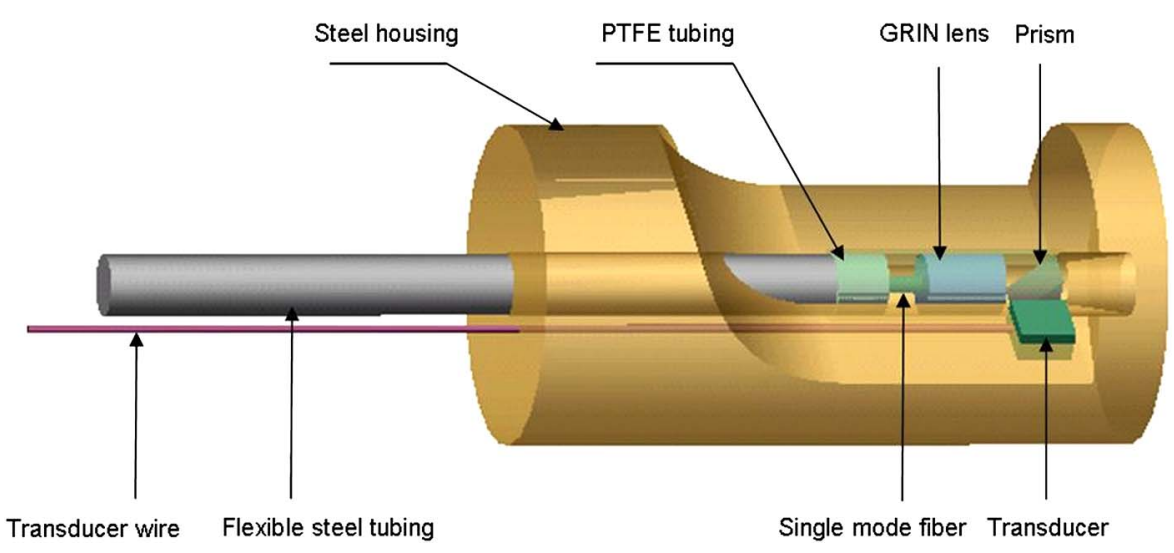

Fig. 1 Schematic of OCT-US probe.

Inc., Orangeburg, SC) with an inner diameter of $0.6 \mathrm{~mm}$ and a wall thickness of $50 \mu \mathrm{m}$. The axial and lateral resolutions of the OCT system with probe are 8 and $20 \mu \mathrm{m}$, respectively. The rest of the bare optical fiber is protected by a 0.51-mm-diam flexible stainless steel tubing, which ensures efficient transmission of rotational torque from the rotary joint to the probe distal end. As for the US part, a single element, unfocused US piezoelectric (PZT) transducer (PZT-5H, $40 \mathrm{MHz}$, aperture area $0.16 \mathrm{~mm}^{2}$ ) was fabricated with a fractional bandwidth of $50 \%$ at $-6 \mathrm{~dB}$. The measured two-way insertion loss for this miniature transducer is $26 \mathrm{~dB}$ at $40 \mathrm{MHz}$ after compensating for attenuation in water, and its theoretical axial and lateral resolutions are approximately 38 and $400 \mu \mathrm{m}$, respectively. The OCT probe and US transducer were fixed side by side in a stainless steel tube housing on which a window was made to allow the light beam and sound wave to exit. The integrated OCT-US probe has a maximum outer diameter of $2.4 \mathrm{~mm}$. To ensure coregistered images, the transducer element was placed next to the exit light beam so that both OCT and US viewed at approximately the same site during imaging.

The OCT-US probe is connected to the OCT-US system as shown in Fig. 2. In the swept-source OCT (SSOCT) system, as reported in Ref. 14, light from a SS (center wavelength, $1310 \mathrm{~nm}$; FWHM bandwidth, $100 \mathrm{~nm}$; output power, $2.7 \mathrm{~mW}$; scanning rate, $20 \mathrm{kHz}$; Santec Corp., Komaki, Aichi, Japan) was split by an $80 / 201 \times 2$ coupler, with $80 \%$ of the power directed to the OCT-US probe and the remaining $20 \%$ to the reference arm. Two circulators were used in both arms to redirect backscattered and backreflected light to the two input ports of a 50/50 $2 \times 2$ coupler for balanced detection. The detected OCT signal was digitized by a 14-bit data acquisition board (National Instrument Corp., Austin, TX) operating at a sampling rate of $33 \mathrm{MHz}$. As for the US part, a two-way pulse-echo measurement was performed using a pulser/receiver (Olympus NDT, Inc., Kennewick, WA). The detected US echo signal was digitized by a 12-bit data acquisition board (Gage Applied Technologies, Lockport, IL) working at the sampling rate of $400 \mathrm{MHz}$. A function generator (Agilent Technologies, Inc., Santa Clara, CA) provided a 2.5-KHz trigger signal to the pulser/receiver and the data acquisition board. Rotational scanning was achieved using a rotational motor and a rotary joint, which consisted of a fiberoptic rotary joint (Princetel, Inc., Pennington, NJ) and a homemade electrical slip ring, to enable transmission of both electrical and optical signals between rotary and stationary parts of the system.

A section of normal rabbit aorta was prepared and fixed in $10 \%$ formalin for $24 \mathrm{~h}$ and then preserved in phosphate buffer. During the experiment, the tissue was pinned to a piece of cork and immersed into saline, allowing simultaneous collection of both OCT and US signals. Rotational speed of the motor was set to 1 revolution/s.

\section{Results and Discussion}

In vitro imaging of normal rabbit aorta was performed using the integrated OCT-US system to demonstrate its feasibility in intravascular imaging, as shown in Fig. 3. It can be clearly indentified that the OCT image [Fig. 3(a)] offers higher reso-

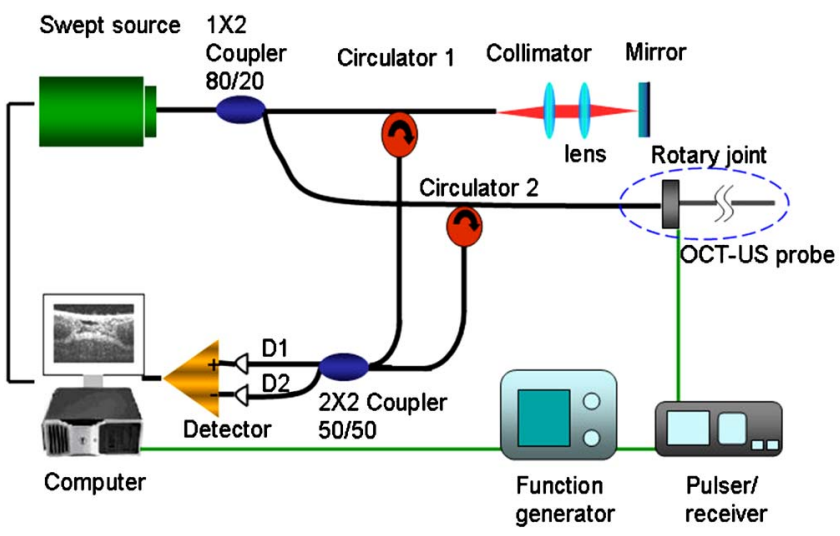

(a)

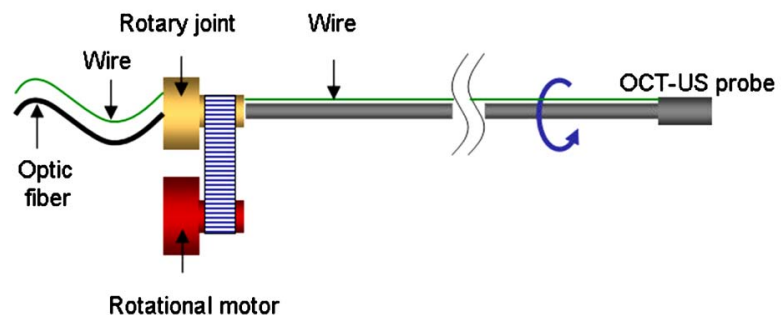

(b)

Fig. 2 Schematic of (a) OCT-US imaging system and (b) enlarged view of rotary joint and probe. 

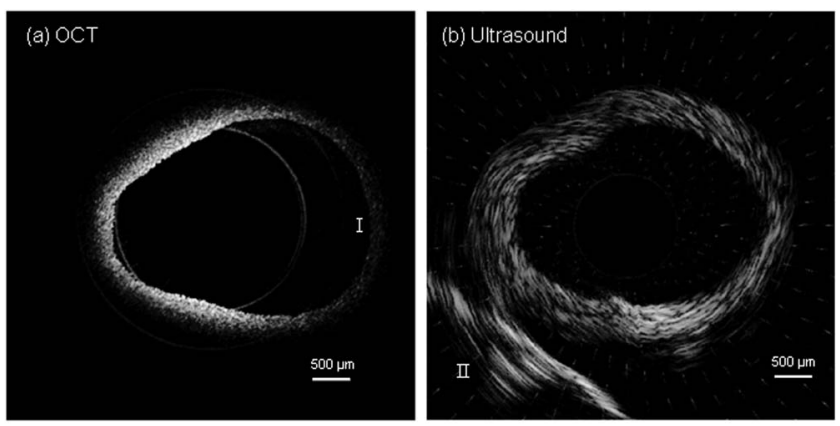

Fig. 3 (a) OCT and (b) US images of rabbit aorta acquired by the dual-modality OCT-US imaging system.

lution than the US image [Fig. 3(b)] and provides more detailed information on tissue structure, which is essential for thickness detection of the thin fibrous cap on a TCFA. However, only about a 500- $\mu$ m-thick maximum depth can be visualized in Fig. 3(a); the penetration depth is even shallower (region I) when the tissue is far away from the probe due to sensitivity roll-off. On the other hand, although its resolution is inferior to that of OCT, the US image [Fig. 3(b)] provides a full-depth cross-sectional image of the aorta-even the cork that was used to position the tissue can be seen (region II). The deep imaging depth is critical for visualizing the large lipid pool within a TCFA, which is one of the important features for plaque characterization. Therefore, the integration of the two imaging modalities can offer complementary information for atherosclerosis diagnosis that cannot be obtained by either modality alone.

The OCT image quality can be further improved by optimizing the OCT system and probe design. Light absorption by water can be reduced by switching to a 1060-nm SSOCT system to achieve a higher signal-to-noise ratio. Moreover, in the current probe design, the OCT probe size is quite small $(\sim 0.5 \mathrm{~mm})$ while its working distance is relatively long $(\sim 3.5 \mathrm{~mm})$ in order to be compatible with the size of the US transducer housing. Thus, there is a trade-off in OCT imaging quality compared to that of a high-quality OCT system. Improved imaging quality can be obtained by minimizing the overall size of the OCT-US probe, which would also provide more flexibility for imaging vessels with smaller dimensions. The periodic noise in the US image [Fig. 3(b)] was caused by electromagnetic interference generated by the motor. An effective electromagnetic shielding strategy is necessary to eliminate this noise. Nevertheless, our current prototype OCT-US probe has been successfully used to acquire OCT and US images simultaneously, demonstrating its possible applications in atherosclerosis detection and diagnosis. Further research will focus on the improvement of probe design, which will ensure optimized imaging quality for both OCT and US, miniaturization and enhanced flexibility of the integrated dual-modality probe that is suitable for in vivo detection of atherosclerotic plaques.

\section{Conclusion}

We have presented a novel OCT-US imaging system for the application of intravascular imaging. The dual-modality probe consists of both OCT optical components and an US transducer, enabling both OCT and US imaging at the same time.
In vitro images of normal rabbit aorta were acquired using this imaging system, which demonstrated its feasibility in intravascular imaging and its potential in detection and characterization of atherosclerotic plaques. Integration of the two imaging modalities combines the unique advantages of OCT and US, which cannot be obtained by using either of them alone; an integrated OCT-US probe can provide both OCT and US imaging simultaneously so that cost and physician's time will be reduced significantly compared to using separate probes.

\section{Acknowledgments}

We thank Hongrui Li and Tanya Burney for preparing the tissue and Wei Wei for his assistance during the experiment. This work is based on research supported by the NIH (Grants No. CA-91717, No. EB-00293, No. EB10090, and No. RR01192) and the U.S. Air Force Office of Scientific Research, Medical Free-Electron Laser Program FA9550-08-1-0384.

\section{References}

1. A. J. Lusis, “Atherosclerosis," Nature 407, 233-241 (2000).

2. F. D. Kolodgie, A. P. Burke, A. Farb, H. K. Gold, J. Y. Yuan, J. Narula, A. V. Finn, and R. Virmani, "The thin-cap fibroatheroma: a type of vulnerable plaque-the major precursor lesion to acute coronary syndromes," Curr. Opin. Cardiol. 16, 285-292 (2001).

3. R. Virmani, A. P. Burke, A. Farb, and F. D. Kolodgie, "Pathology of the unstable plaque," Prog. Cardiovasc. Dis. 44, 349-356 (2002).

4. G. Pasterkamp, E. Falk, H. Woutman, and C. Borst, "Techniques characterizing the coronary atherosclerotic plaque: Influence on clinical decision making," J. Am. Coll. Cardiol. 36, 13-21 (2000).

5. B. N. Potkin, A. L. Bartorelli, J. M. Gessert, R. F. Neville, Y. Almagor, W. C. Roberts, and M. B. Leon, "Coronary-artery imaging with intravascular high-frequency ultrasound," Circulation 81, 15751585 (1990).

6. L. Landini and L. Verrazzani, "Spectral characterization of tissues microstructure by ultrasounds-a stochastic approach," IEEE Trans. Ultrason. Ferroelectr. Freq. Control 37, 448-456 (1990).

7. S. W. E. van de Poll, C. L. de Korte, A. F. W. van der Steen, G. J. Puppels, and A. van der Laarse, "Coronary atherosclerotic plaque characterization using IVUS elastography and Raman spectroscopy," in 2000 IEEE Ultrasonics Symp. Proc. An Int. Symp. Cat. No. 00CH37121. (2000).

8. D. Huang, E. A. Swanson, C. P. Lin, J. S. Schuman, W. G. Stinson, W. Chang, M. R. Hee, T. Flotte, K. Gregory, C. A. Puliafito, and J. G. Fujimoto, "Optical coherence tomography," Science 254, 1178-1181 (1991).

9. M. U. Farooq, A. Khasnis, A. Majid, and M. Y. Kassab, "The role of optical coherence tomography in vascular medicine," Vasc. Med. 14, 63-71 (2009).

10. G. J. Tearney, I. K. Jang, and B. E. Bouma, "Optical coherence tomography for imaging the vulnerable plaque," J. Biomed. Opt. 11, 021002-1-021002-10 (2006).

11. M. E. Brezinski, G. J. Tearney, B. E. Bouma, J. A. Izatt, M. R. Hee, E. A. Swanson, J. F. Southern, and J. G. Fujimoto, "Optical coherence tomography for optical biopsy-properties and demonstration of vascular pathology," Circulation 93, 1206-1213 (1996).

12. T. Sawada, J. Shite, H. M. Garcia-Garcia, et al., "Feasibility of combined use of intravascular ultrasound radiofrequency data analysis and optical coherence tomography for detecting thin-cap fibroatheroma," Eur. Heart J. 29, 1136-1146 (2008).

13. M. Kawasaki, B. E. Bouma, J. Bressner, S. L. Houser, S. K. Nadkarni, B. D. MacNeill, I. K. Jang, H. Fujiwara, and G. J. Tearney, "Diagnostic accuracy of optical coherence tomography and integrated backscatter intravascular ultrasound images for tissue characterization of human coronary plaques," J. Am. Coll. Cardiol. 48, 81-88 (2006).

14. J. P. Su, J. Zhang, L. F. Yu, H. G. Colt, M. Brenner, and Z. P. Chen, "Real-time swept source optical coherence tomography imaging of the human airway using a microelectromechanical system endoscope and digital signal processor," J. Biomed. Opt. 13, 030506-1030506-3 (2008) 